\title{
Fouilles archéologiques à Serra del Cedro et Civita di Tricarico
}

Campagne 2020

Stéphane Bourdin et Olivier de Cazanove

\section{CpenEdition}

Journals

Édition électronique

URL : https://journals.openedition.org/baefe/3643

DOI : $10.4000 /$ baefe.3643

ISSN : 2732-687X

Éditeur

ResEFE

Référence électronique

Stéphane Bourdin, Olivier de Cazanove, «Fouilles archéologiques à Serra del Cedro et Civita di Tricarico » [notice archéologique], Bulletin archéologique des Écoles françaises à l'étranger [En ligne], Italie, mis en ligne le 23 juillet 2021, consulté le 26 juillet 2021. URL : http://journals.openedition.org/ baefe/3643 ; DOI : https://doi.org/10.4000/baefe.3643

Ce document a été généré automatiquement le 26 juillet 2021.

\section{cc) (†) $\odot$}

Le Bulletin archéologique des Écoles françaises à l'étranger est mise à disposition selon les termes de la Licence Creative Commons Attribution - Pas d'Utilisation Commerciale - Pas de Modification 4.0

International. 


\title{
Fouilles archéologiques à Serra del Cedro et Civita di Tricarico
}

\author{
Campagne 2020
}

Stéphane Bourdin et Olivier de Cazanove

\section{NOTE DE L'AUTEUR}

Date précise de l'opération : 10-27 août 2020

Autorité nationale présente : MIBACT ; Soprintendenza Archeologia Belle Arti Paesaggio della Basilicata.

Numéro de mission : Concession DG-ABAP|31/07/2020|DECRETO 1060

Composition de l'équipe de terrain : Stéphane Bourdin université Lyon II, directeur de la fouille, UMR 5189 HiSoMA ; Olivier de Cazanove, université Paris 1, directeur scientifique, UMR 7041 ARSCAN ; Lucas Aniceto, chercheur postdoctoral, Labex PasP, UMR 7041 ARSCAN ; Hugo Blanchet, chercheur postdoctoral, Université Bordeaux Montaigne ; Charlotte Boyer, doctorante, UMR 7041 ARSCAN, anthropologue ; Alberto Brutto, master, Paris 7 ; Marion Claude, chercheuse postdoctorale, Labex Hastec, UMR 8210 ANHIMA ; Baptiste Gouttenègre, master, Lyon 2 ; Zélie Laurendeau, master, Paris 1 ; Christina Popova, master, Lyon 2 ; Clément Salviani, doctorant UMR 7041 et ATER Bordeaux Montaigne ; Thomas Terrasse, topographe (TERRA-H) ; Corentin Voisin, doctorant, univ. Strasbourg ; Euan Wall, chargé d'études et de recherche, INHA.

Établissements porteurs de l'opération : EFR, Université Paris I Panthéon Sorbonne, Université Lumière Lyon 2, CNRS

1 Malgré la crise sanitaire, les campagnes de fouilles sur les deux villes fortifiées de Serra del Cedro et Civita di Tricarico ont pu se dérouler normalement, dans le cadre du programme Ignobilia oppida Lucanorum / Les places fortes sans renom des Lucaniens soutenu par l'École française de Rome en partenariat avec la Surintendance de Basilicate. En revanche, les campagnes d'étude de mobilier n'ont pas eu lieu en raison de la pandémie. On a du moins pu contribuer à la documentation pour le volume Civita di 
Tricarico III (en préparation) en complétant la batterie de datations ${ }^{14} \mathrm{C}$ de l'entrepôt : le chien trouvé dans la pièce 1 a été consommé (étude de S. Lepetz) entre 371 et 199 (fourchette $2 \Sigma$ Beta Analytic). On dispose désormais de 7 datations ${ }^{14} \mathrm{C}$ de l'incendie de l'entrepôt. Leur combinaison (à $1 \Sigma$ ) donne comme dates les plus probables de cet incendie : 297, 276-275 ou 260-259 av. J.-C.

\section{Serra del Cedro}

\section{La fouille dans le secteur central}

2 Au centre de l'habitat, entre le sommet principal (Tempa dell'Altare, alt. $858 \mathrm{~m}$ ) et le sommet secondaire où se trouve la maison Alpha (alt. $817 \mathrm{~m}$ ), s'étend un vallon, orienté vers l'est, et traversé par un chemin de terre abusif, récemment tracé. Sur les bas-côtés de ce chemin sont visibles de nombreux tessons, des fragments de tuiles plates et d'imbrices, ainsi que quelques fragments de décorations architectoniques, rejetés par les engins mécaniques.

3 Au sommet du vallon, un alignement de blocs affleurant a été nettoyé, ce qui a permis de découvrir un mur (US 277), épais de 0,85 m, que l'on a suivi sur 4,30 $\mathrm{m}$ de long. Ce mur, orienté $\mathrm{N} / \mathrm{s}$, délimitait probablement une habitation. De gros blocs équarris $(0,60 \times 0,30 \mathrm{~m}$ et $0,60 \times 0,38 \mathrm{~m})$, en position de chute dans la pente, proviennent des assises de ce mur. Lors de la prochaine campagne, on cherchera à délimiter précisément les dimensions et la nature de cet édifice (fig. 1).

Fig. 1. Serra del Cedro : Mur 277 au sommet du vallon.

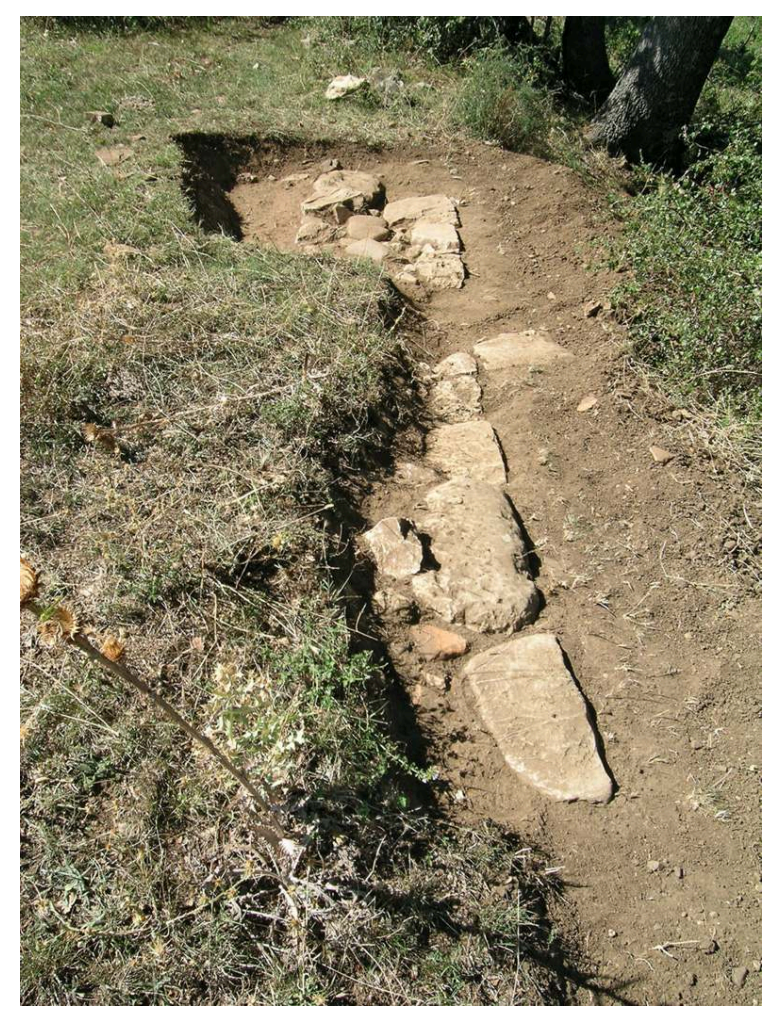

S. Bourdin. 


\section{La fouille de la maison Alpha}

4 La maison Alpha est une grande maison à pastas, avec trois pièces $(2,3,4)$ qui s'ouvrent sur une grande pastas rectangulaire (pièce 1), ouverte par un stylobate, au centre duquel s'élevait une colonne. Les pièces 3 et 4 sont rectangulaires et de dimensions similaires $\left(28,3 \mathrm{~m}^{2}\right.$ et $\left.27,54 \mathrm{~m}^{2}\right)$; la pièce 2 est en revanche carrée $\left(20,16 \mathrm{~m}^{2}\right)$. La pastas mesure 83,72 $\mathrm{m}^{2}$. Deux ailes se développent à l'est de la pastas, avec les pièces 6 et 7 sur le côté nord et l'ensemble de pièces 5 a (pièce rectangulaire, 21,28 $\mathrm{m}^{2}$ ), b (pièce carrée, $6,9 \mathrm{~m}^{2}$ ) et c (carrée, $7 \mathrm{~m}^{2}$ ) sur le côté sud, l'ensemble délimitant une vaste cour trapézoïdale $\left(10 \times 8=80 \mathrm{~m}^{2}\right)$. L'élévation des murs est bien conservée, sur une dizaine d'assises : de 0,48 à 1,10 m de hauteur pour le mur 144 et $1,07 \mathrm{~m}$ de hauteur pour le mur 161 (fig. 2).

Fig. 2. Serra del Cedro : Vue par drone de la maison Alpha.

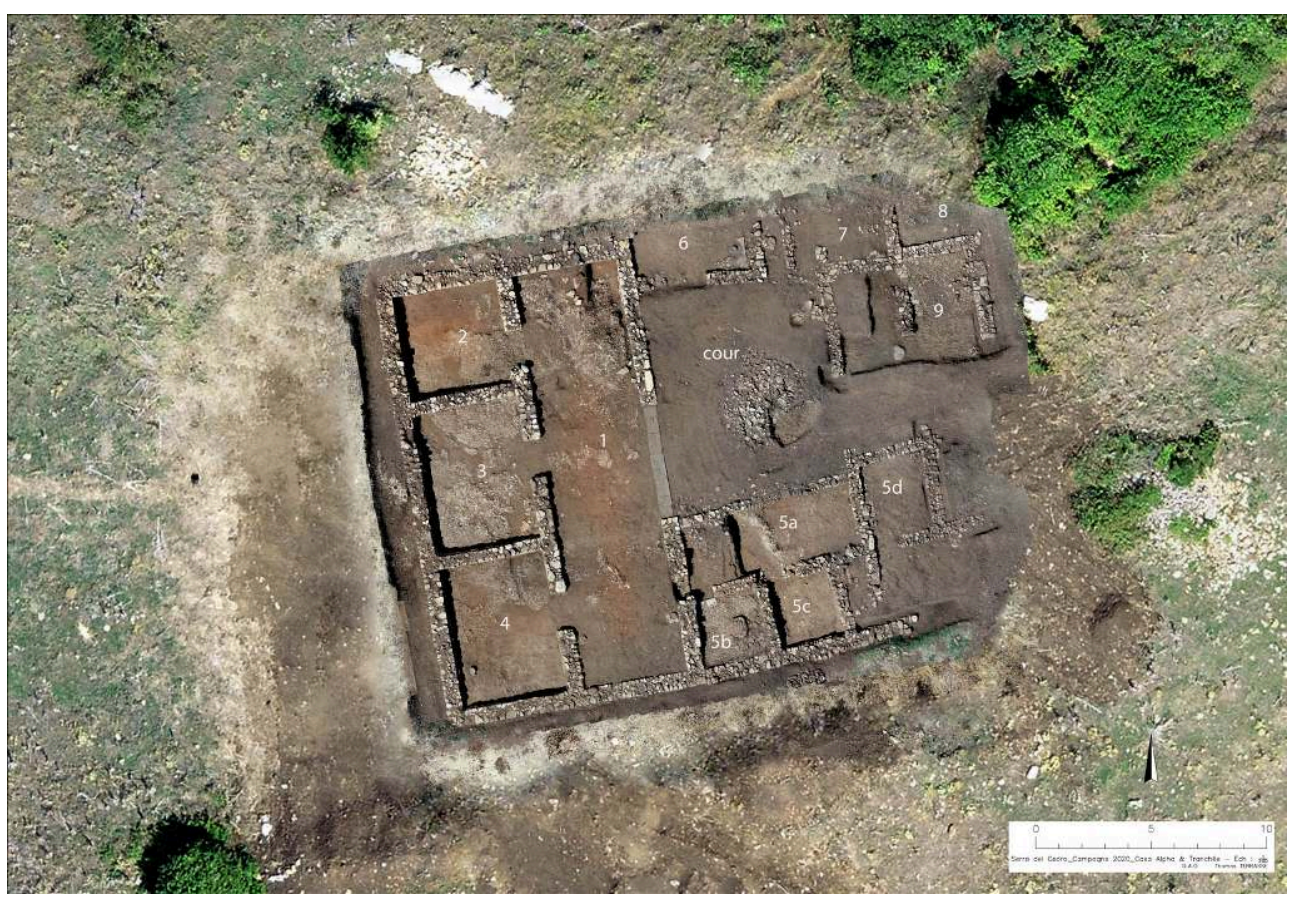

Rel. T. Terrasse ; DAO S. Bourdin.

5 La pièce 4 a été fouillée jusqu'au sol, en démontant un niveau d'écroulement (US 258), argilo-limoneux, avec de nombreux fragments de tegulae et d'imbrices, et des tessons écrasés à plat sur le sol. Ce sol, comme dans les pièces 2 et 3 , est en partie taillé dans la roche naturelle. L'US 258 a livré une quantité importante de fragments de vases à vernis noir (skpyhoi, coupes, assiettes), de céramique achrome ou à bandes peintes (dont plusieurs fragments d'ollae), un certain nombre de fragments de vases de cuisson, ainsi qu'une quarantaine d'esquilles d'os animaux (fig. 3). 
Fig. 3. Serra del Cedro : Maison Alpha, pièce 4.

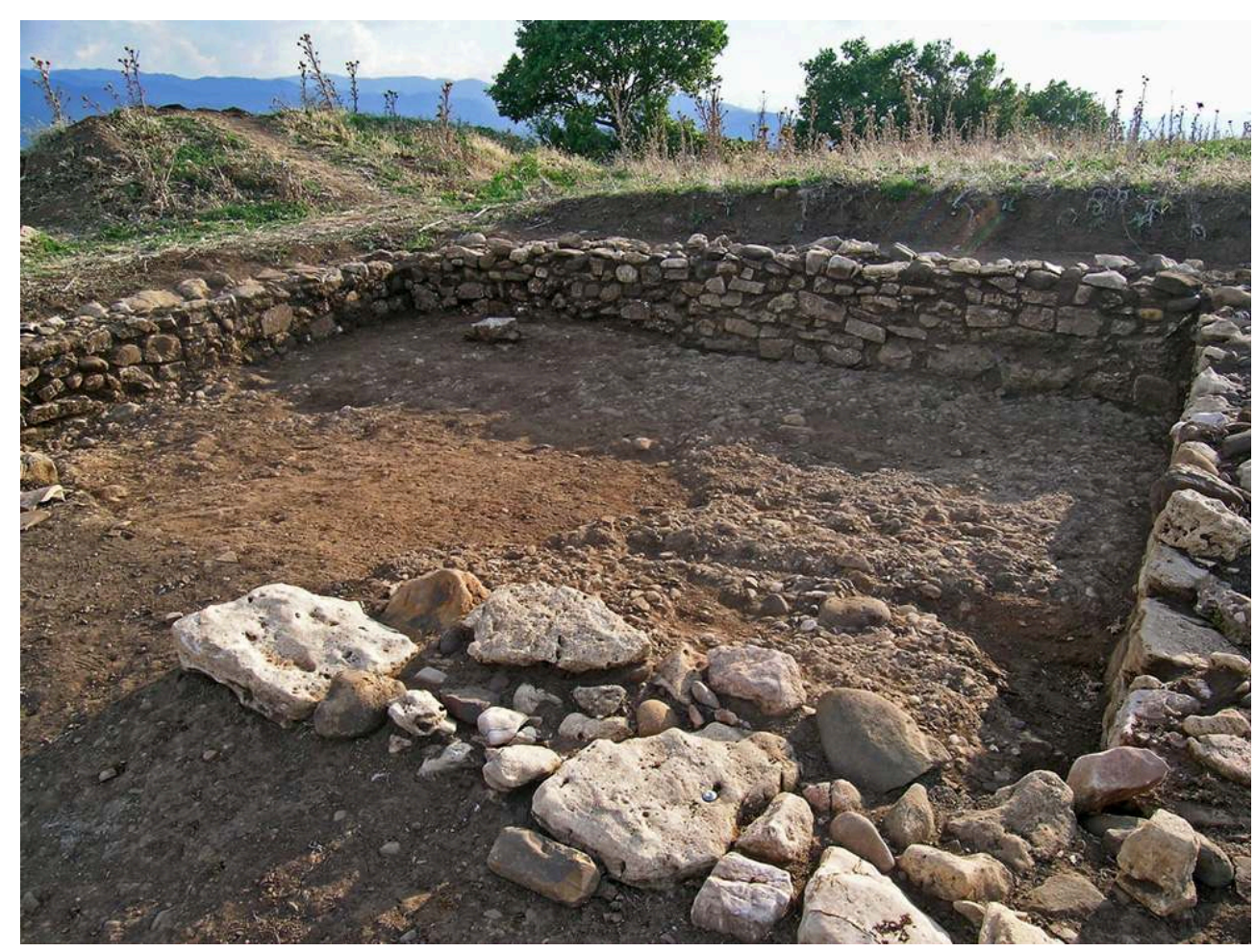

S. Bourdin.

6 La pièce 3 a également été entièrement fouillée, jusqu'au niveau du sol, formé pour la plus grande part par la roche naturelle retaillée. Ce niveau de sol a été atteint après le démontage d'une couche homogène de démolition, formée, dans sa partie supérieure, d'un très faible niveau d'écroulement de tuiles et de quelques blocs tombés des assises supérieures du mur 161. Le long du mur 161, on a mis en évidence, comme dans la pièce 2 , la présence d'une petite tranchée, creusée dans la roche, au fond de laquelle était posée la première assise du mur lui-même.

7 Cette couche (US 273) a livré en majorité des fragments de céramique achrome et de cuisine, ainsi que quelques fragments de céramique à vernis noir, deux fragments de dolium et 5 pesons de métier à tisser. Les trois pièces $(2,3,4)$ semblent donc avoir été utilisées pour des activités domestiques, sans que l'on puisse, à ce stade, identifier d'usage particulier. Au niveau du sol de la pièce 3, le long des murs 163 et 145, on a découvert respectivement une olla et une coupe écrasées, tandis que dans l'angle formé par les murs 145 et 164, une série de tuiles plates posées sur le sol forment une petite banquette. Le long du mur 145 gisait la lame d'un gros couteau de fer. Le mur 164, comme le mur parallèle (163), est construit en élévation sur un petit ressaut taillé dans la roche.

8 Dans la partie méridionale de la pastas, le niveau de sol se trouvait sous une couche de démolition homogène (274), qui contenait quelques fragments de tuiles, un certain nombre de fragments de vases à vernis noir, achromes ou de cuisine et 3 pesons de métier à tisser. Les quatre pièces principales de la maison Alpha ont donc été fouillées jusqu'au niveau de circulation. Le sol de la pastas était lui aussi formé en partie par la roche naturelle taillée à l'horizontale et en partie en terre battue. Dans l'angle NE de la pastas (à l'angle des murs 142 et 144), on a réalisé un sondage qui a mis en lumière 
l'abaissement progressif du banc rocheux dans cette zone, jusqu'à une profondeur de $0,58 \mathrm{~m}$ : cette irrégularité de la roche naturelle a rendu nécessaire l'accumulation d'un sédiment rougeâtre compact (270), riche de cailloutis et de fragments de tuiles (provenant donc d'édifices antérieurs à la maison Alpha), qui a rempli cette espèce de tranchée de fondation du mur 142. Parmi le rare matériel (dans la partie supérieure de l'US 270), qui permettrait de dater le remplissage de la tranchée (et la construction des murs 142 et 144), on trouve des fragments de skyphos à vernis noir et achrome.

Dans le prolongement du noyau de la maison Alpha se trouvent les pièces 6 et 7. Entre les deux pièces, un étroit ambitus (largeur : 0,98 m) permettait d'accéder à la cour de la maison. La couche superficielle de l'ambitus (290) a été ôtée, mettant en lumière des portions d'un niveau d'écroulement contenant notamment des fragments de dolium.

10 Sur le côté oriental de la cour, les murs $170,169 / 248$ et 264 (que l'on suit sur une longueur de 3,90 $\mathrm{m}$ et qui était large de $0,63 \mathrm{~m}$ ) forment une grande pièce, divisée en deux nefs par un mur-bahut $(294 ; 2,12 \mathrm{~m} \times 0,60 \mathrm{~m})$. Le mur périmétral méridional de cet espace n'a pas encore été repéré. Le sol en opus spicatum, fait de petits galets, a été complètement dégagé. Il se trouve dans la partie orientale de cette pièce, à l'est du mur 294, tandis que la nef occidentale présente un sol en terre battue. Une grosse lacune de forme ovale au centre du sol suggère la présence d'une vasque ; l'utilisation de l'eau dans la pièce est également indiquée par le pendage $\mathrm{O} / \mathrm{E}$ du sol, qui permettait à l'eau de confluer vers une petite canalisation, formée d'un imbrex retourné, posé en travers du mur 264 (fig. 4).

Fig. 4. Serra del Cedro : Maison Alpha, sol en opus spicatum dans la pièce 9.

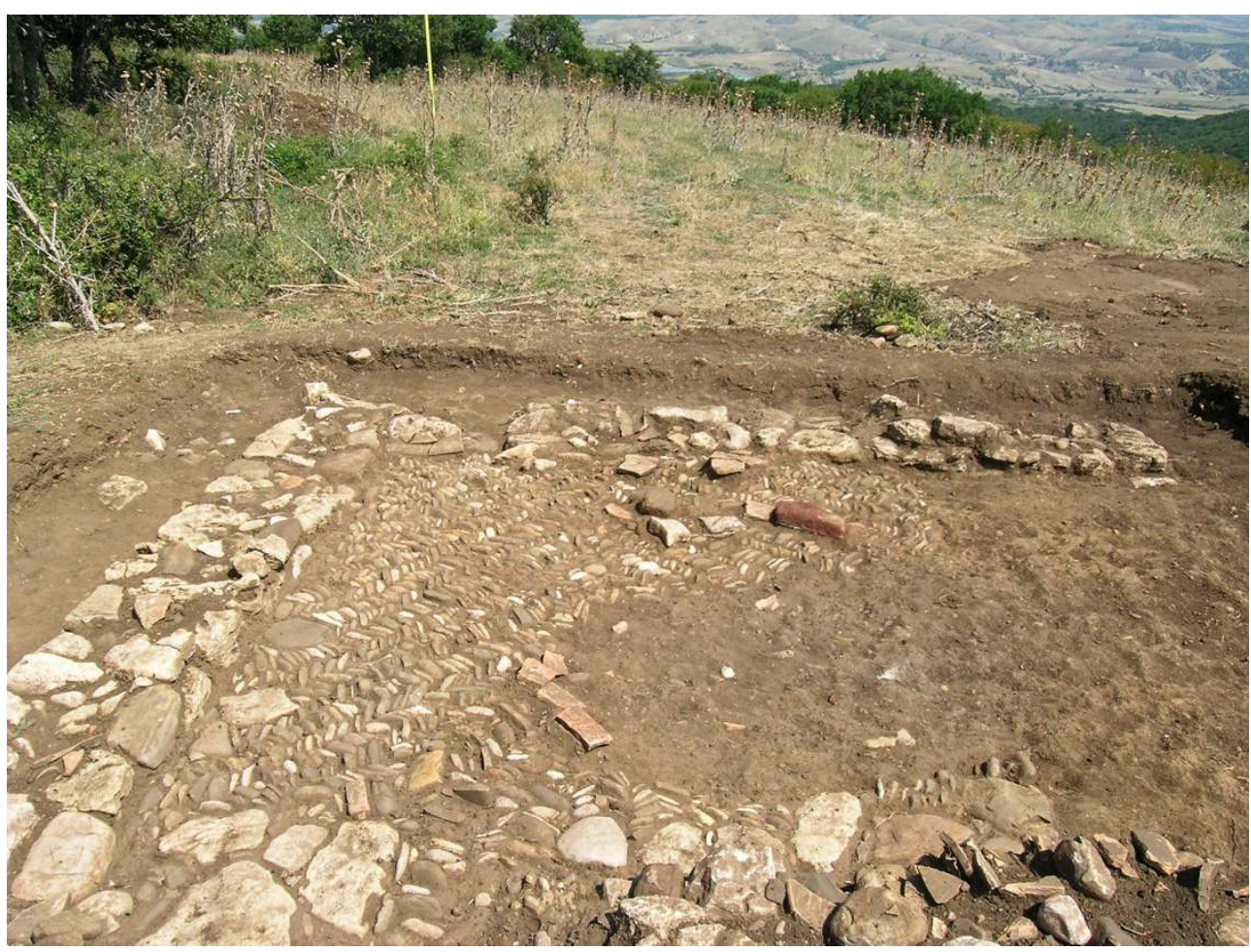

S. Bourdin.

11 L'aile 5 s'ouvre sur la pastas par un seuil, créé dans le mur 142 en le démontant jusqu'à la première assise, qui forme une marche permettant d'accéder à la pastas; cette 
marche se trouve à la même cote que le seuil de la pièce $5 \mathrm{~b}$. Les murs de l'aile 5 sont moins épais que ceux du noyau initial de la maison Alpha, avec une largeur moyenne de $0,50 \mathrm{~m}$ (0,50 m pour le mur 218, 0,53 m pour le mur 251). Ces derniers, comme tous les autres murs de la maison, incorporent du matériel de réemploi (tuiles plates, un fragment de loutérion dans le mur 224). En deux occasions (murs 142 et 224), des tuiles plates ont été disposées dans la partie supérieure du mur, pour créer un lit de pose plan, correspondant peut-être à une réparation. L'aile 5 est subdivisée en trois pièces : une pièce rectangulaire ( $5 \mathrm{a}$ ) et deux cellules carrées (b et c). Un seuil, à l'est, ouvre sur un espace dont on n'a pas encore découvert les limites, mais qui a été, dans un deuxième temps, divisé par le mur 287 (largeur : 0,55 m), dans l'axe du mur oriental (mais légèrement en biais) de la pièce $5 \mathrm{a}(251)$, avec lequel il est chaîné.

Les trois pièces $5 \mathrm{a}$, $\mathrm{b}$ et $\mathrm{c}$ ont été fouillées jusqu'au niveau du sol, qui est en partie retaillé dans la roche naturelle (dans les pièces $5 \mathrm{a}$ et $5 \mathrm{c}$ ). La pièce $5 \mathrm{a}$ présentait un niveau d'écroulement de tuiles peu dense, qui recouvrait un niveau d'abandon. Cette couche (279), riche de matériel céramique, a livré entre autres un fragment de cratère à figures rouges, de la céramique à vernis noir (skyphoi, assiettes), une grande quantité de céramique achrome en argile dépurée, de la céramique de cuisine et deux pesons de métier à tisser. La même situation se retrouve dans la pièce $5 \mathrm{c}$, avec des portions de niveau d'écroulement de tuiles le long du mur 252. Dans le mur 166, deux blocs carrés en grès ( $\mathrm{H}: 0,27$ et $0,28 \mathrm{~m})$, distants de 2,14 $\mathrm{m}$ l'un de l'autre, font penser à un premier aménagement comme portique, avec deux colonnes, qui s'ouvrait par une baie sur la cour. L'espace entre les deux blocs a été fermé dans un second temps. La couche superficielle 275 , dans la pièce $5 \mathrm{c}$, a livré l'habituelle céramique à vernis noir et achrome, de la céramique de cuisine, deux pesons et des esquilles d'os et des dents d'animaux.

13 La pièce $5 \mathrm{~b}$ s'ouvre par un seuil dans l'angle nord-ouest. Deux dalles plates $(0,38 \times 0,10$ et $0,38 \times 0,13 \mathrm{~m}$ ) forment ce seuil et marquent le niveau du sol de la pièce, en opus spicatum de petits galets (292). Ce sol remonte légèrement au niveau des murs 167 et 142, et s'interrompt avant le contact avec les murs 224 et 252. Dans la partie SE de la pièce, on aperçoit la partie inférieure d'un niveau d'écroulement (291), posé directement sur le sol, et un foyer (283), construit avec des fragments de tuiles et de forme globalement rectangulaire $(0,85 \times 0,73 \mathrm{~m})$. Ce foyer était isolé du sol par une couche de terre de $10 \mathrm{~cm}$ d'épaisseur. Quelques inclusions charbonneuses sont visibles à la surface du foyer et une coupelle concavo-convexe a été découverte directement posée sur le sol de la pièce (fig. 5). 
Fig. 5. Serra del Cedro : Maison Alpha, sol en opus spicatum et foyer dans la pièce 5b.

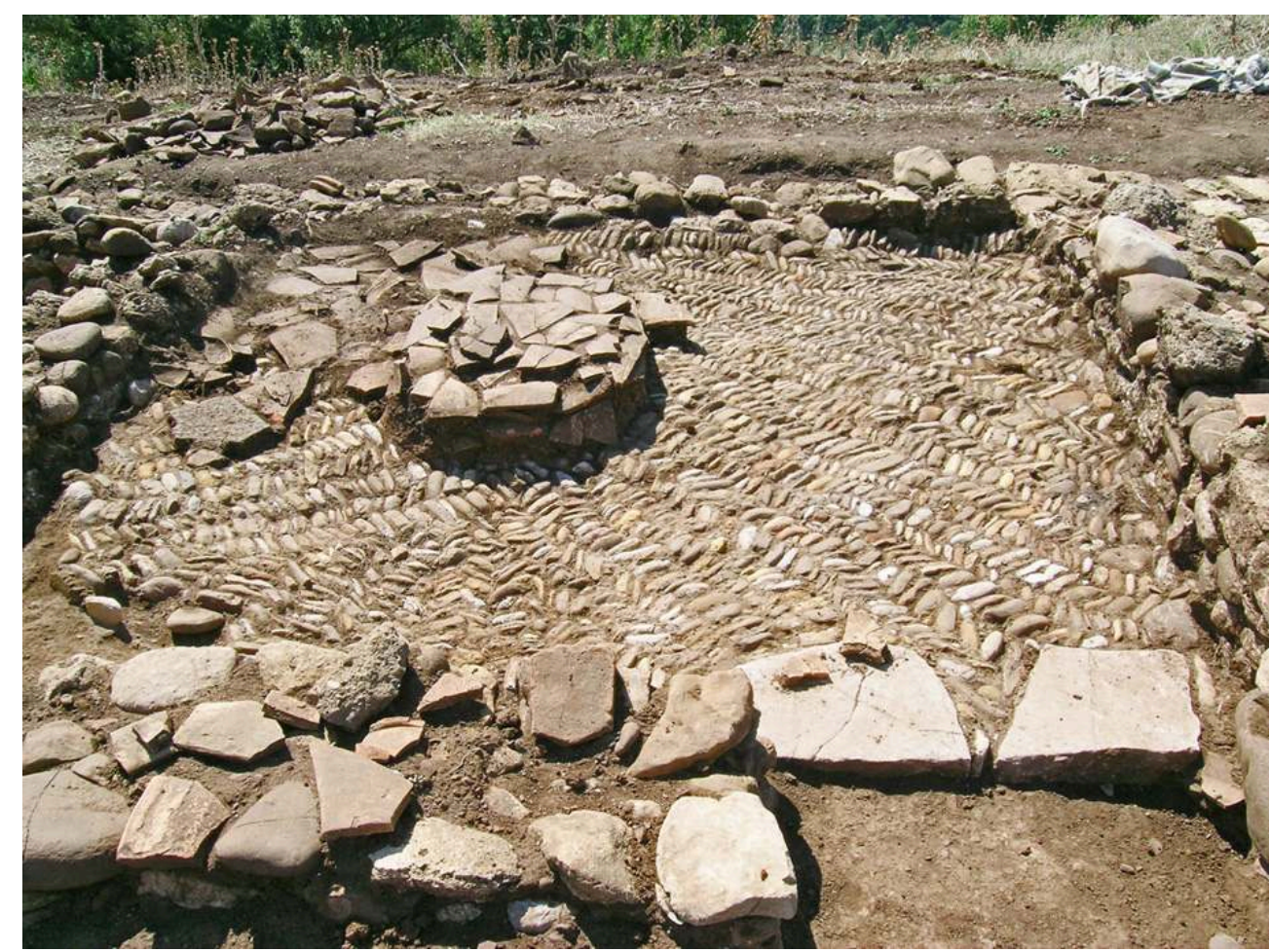

S. Bourdin

14 À l'est de l'aile 5, la situation n'est pas encore très claire. Le mur 167 s'interrompt sans qu'on comprenne si l'espace délimité par les murs 218, 167 et 224/266 était fermé ou non. Dans cet espace s'ouvre une petite pièce carrée $\left(5 \mathrm{~d} ; 9,01 \mathrm{~m}^{2}\right)$, délimitée par les murs 251, 253, 261 et 266, dans laquelle on entrevoit un niveau d'écroulement de tuiles peu dense. La fonction de cette pièce n'est pas encore définie ; ses murs sont construits selon un module léger (largeur $0,50 \mathrm{~m}$ ). À l'est de la pièce $5 \mathrm{~d}$, le mur 296, qui prolonge 266 et qui est large de $0,55 \mathrm{~m}$, a été suivi pour l'instant sur une longueur de $1,80 \mathrm{~m}$; sa présence laisse supposer l'existence d'une autre pièce, dont le mur périmétral nord fermerait l'espace à deux nefs et au sol en opus spicatum. Il reste à éclaircir la question de l'ouverture de la cour : s'ouvre-t-elle sur une large entrée, entre les murs 170 et 251, ou n'y accédait-on qu'à travers l'étroit ambitus entre les pièces 6 et 7 au nord?

15 Les murs 142 (mur est de la pastas), 166 (mur périmétral nord de la pièce $5 \mathrm{a}$ ) et 170 délimitent une cour trapézoïdale, d'environ $80 \mathrm{~m}^{2}$. On a dégagé l'humus superficiel qui recouvrait la cour (289), jusqu'au niveau d'arasement des murs, et on a mis en évidence la présence, au centre de la cour, d'un gros pierrier (281), de forme ovale $(4,40 \mathrm{x}$ $2,80 \mathrm{~m}$ ). Ce pierrier se présente comme le remplissage d'une fosse, avec un sédiment noirâtre friable, qui enserre de nombreux blocs de calcaire, de grès et de poudingue, de module varié $(0,10$ à $0,50 \mathrm{~m})$, des fragments d'imbrices, de tegulae, de dolia et d'un loutérion, ainsi qu'une paroi de terre cuite avec une décoration plastique, qui correspond peut-être à une paroi de baignoire ou d'une margelle de puits. Les parois de la fosse descendent jusqu'à une profondeur de $30 \mathrm{~cm}$ environ, où on rencontre un sédiment plus compact (299). 
Lors de la prochaine campagne, nous prévoyons d'achever la fouille de la cour, de la pièce à deux nefs et d'élargir la zone de fouille vers l'est pour délimiter complètement la maison Alpha dans son ensemble.

\section{Civita di Tricarico}

17 Après les prospections géophysiques menées en 2019 à Civita di Tricarico, on a complété en 2020 le transect (fig. 6) traversant le quartier 3M (celui de la maison du Monolithe, de la maison $\mathrm{M}$ et de la maison des Moules) pour en étudier l'organisation et les limites. Au nord du temple P (fouillé en 2003 et 2005), de l'entrepôt R (fouillé en 2002-2003) et de la maison Z1 (fouillée en 2018), la maison Z2 a été en partie mise au jour cette année.

Fig. 6. Civita di Tricarico : secteurs et tranchée fouillés en 2020.

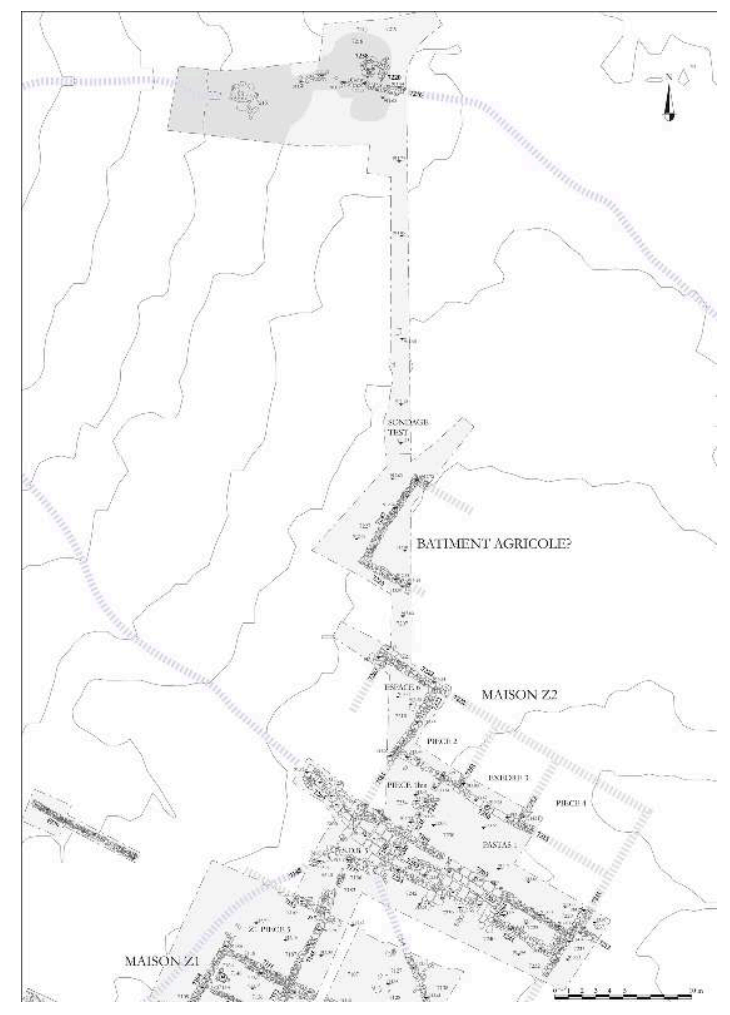

Rel. T. Terrasse ; DAO O. de Cazanove.

18 Au-delà de la maison $\mathrm{Z2}$, le transect a été prolongé par une tranchée exactement orientée sud-nord, longue de $58 \mathrm{~m}$, destinée à étudier l'articulation entre espaces bâtis non bâtis (ces derniers révélés par les prospections géophysiques, dès 2007-2008). La tranchée 2020 n'a de fait mis en évidence qu'une structure légère, cabane ou enclos, puis des espaces vides, jusqu'à une canalisation alimentée par un puits à chadouf. On vérifie ainsi l'alternance de bandes bâties (le quartier 3M a $65 \mathrm{~m}$ de large en moyenne) et de terrains à vocation agricole ou agro-pastorale. 


\section{Avant la maison Z2 : la maison Z1 étendue (première phase)}

l'entrepôt R. Elle était séparée de lui par un ambitus et un mur continu. Pour cette raison, elle s'ouvrait vers l'est (et non vers le sud, comme les autres maisons). Pour cette raison aussi, elle n'avait pu s'étendre que vers le nord. De ce côté, on avait mis partiellement au jour une pièce 5, d'env. 4,80 x 5,10 m, limitée à l'est par le mur 7145, dans le prolongement du mur nord-sud (7113) de Z1. Le même mur se prolongeait encore au-delà du mur nord (7155) de la pièce 5 , avant de disparaître sous le dallage 7160 qu'on avait commencé à mettre en évidence en limite de sondage. Il restait donc à poursuivre la fouille en mettant plus largement au jour cette maison Z1 «étendue». Il restait aussi à comprendre - et cette question n'est pas encore entièrement résolue - comment et dans quel sens s'était agrandie l'habitation :

- du sud au nord, à partir de la maison Z1 presque carrée fouillée en 2018 ;

- du nord au sud, à partir d'un premier corps de bâtiment situé sous la maison Z2 ;

- ou par la réunion de deux maisons à l'origine distinctes ;

- ou enfin en supposant que la maison Z1 «étendue » a occupé (dès l'origine ?) deux lots (ou plutôt un lot et demi, le demi-lot au sud de la maison carrée Z1 étant occupé par l'entrepôt R).

Quoi qu'il en soit, il est clair que la maison Z2 est postérieure à « Z1 étendue ». En effet :

- la prolongation du mur 7145 vers le nord passe sous le dallage 7160 qui appartient, comme on le verra, à une extension de la maison $\mathrm{Z2}$;

- l'extrémité nord (7223) du mur 7181 qui forme le côté ouest de la maison Z2 est alignée comme le mur 7113-7145. On pourrait proposer d'y voir un tronçon de mur de première phase réutilisé dans la seconde, avec un raccord très visible ;

- enfin et surtout, un mur 7224-7241, arasé et interrompu par le dallage 7202, à 1,1/2 m en avant de la façade de Z2, avec une direction nettement différente, doit appartenir à la première phase, dont il suit le système d'orientation.

21 À ce stade de l'enquête, alors qu'à l'intérieur de la maison Z2 les niveaux de démolition de tuiles n'ont pas été démontés, il n'est pas encore possible de proposer une hypothèse de restitution planimétrique précise pour le bâtiment sous-jacent dont l'existence est cependant assurée.

Un problème complexe de stratigraphie horizontale est posé par l'angle sud-est de la maison Z2. Il englobe un angle en sens inverse (nord-ouest) formé par deux murs d'équerre $7211 \mathrm{~b}$ et 7212 , qui devait être pertinent à un autre bâtiment de première phase.

\section{La maison Z2 (deuxième phase de l'occupation du secteur)}

On en connaissait l'existence et l'emplacement depuis la prospection géophysique (électrique) de 2007, qui avait également permis de prédire qu'il s'agissait d'une maison à pastas élémentaire typique, comparable (à Civita di Tricarico) à la maison des moules, aux maisons $\mathrm{M}, \mathrm{W}, \mathrm{Y} 1,2,6$ et 7 et (à Serra del Cedro) à la maison Alpha ${ }^{1}$. La fouille a toutefois permis de préciser et de corriger sur certains points l'analyse préliminaire qui en avait été faite ${ }^{2}$. 
24 proche de $11 \mathrm{~m}=40$ pieds osques de $27,5 \mathrm{~cm}$, profondeur standard des maisons de Civita di Tricarico). La maison $\mathrm{Z} 2$ atteint cependant $22,73 \mathrm{~m}$ de longueur totale est-ouest car le mur du fond (7222) se prolonge au-delà du renfort d'angle nord-ouest, bien visible, de l'habitation. Un espace (6) est donc accolé, à l'ouest, au bloc central de la demeure. Il ne s'agit toutefois pas d'un ajout, comme on verra, mais plutôt d'un espace antérieur qui a été incorporé tant bien que mal à la maison Z2. L'existence de deux phases distinctes, et d'un raccord assez mal réalisé entre les deux, se voit en tout cas nettement, au niveau du mur ouest (7223-7181) de la pièce 2, qui paraît comme tordu (voir plus haut). (dim. int. 17,2 x 4,5 m) et ce n'est qu'ensuite qu'elle sera divisée en deux parties inégales par une cloison transversale (7235) (fig. 7). La pastas s'ouvre sur l'extérieur, au sud, par un seuil (7203) large de 4,40-4,45 m, profond de $70 \mathrm{~cm}$ au max., composé de cinq dalles de grès finement piqueté. Une trace ancienne, oblique, sur l'une d'elles, doit correspondre à un sillon de charrue. La dalle du milieu est la plus large $(1,15 \times 0,7 \mathrm{~m})$; il est possible qu'elle ait supporté une colonne centrale, à moins qu'il y en ait eu deux, disposées en ce cas sur les dalles 2 et 4 . Le seuil de la pastas n'est exactement centré, ni par rapport à la façade de la maison, ni par rapport au seuil de l'exèdre dont on parlera dans un instant.

Fig. 7. Civita di Tricarico : la maison Z2 en cours de fouille.

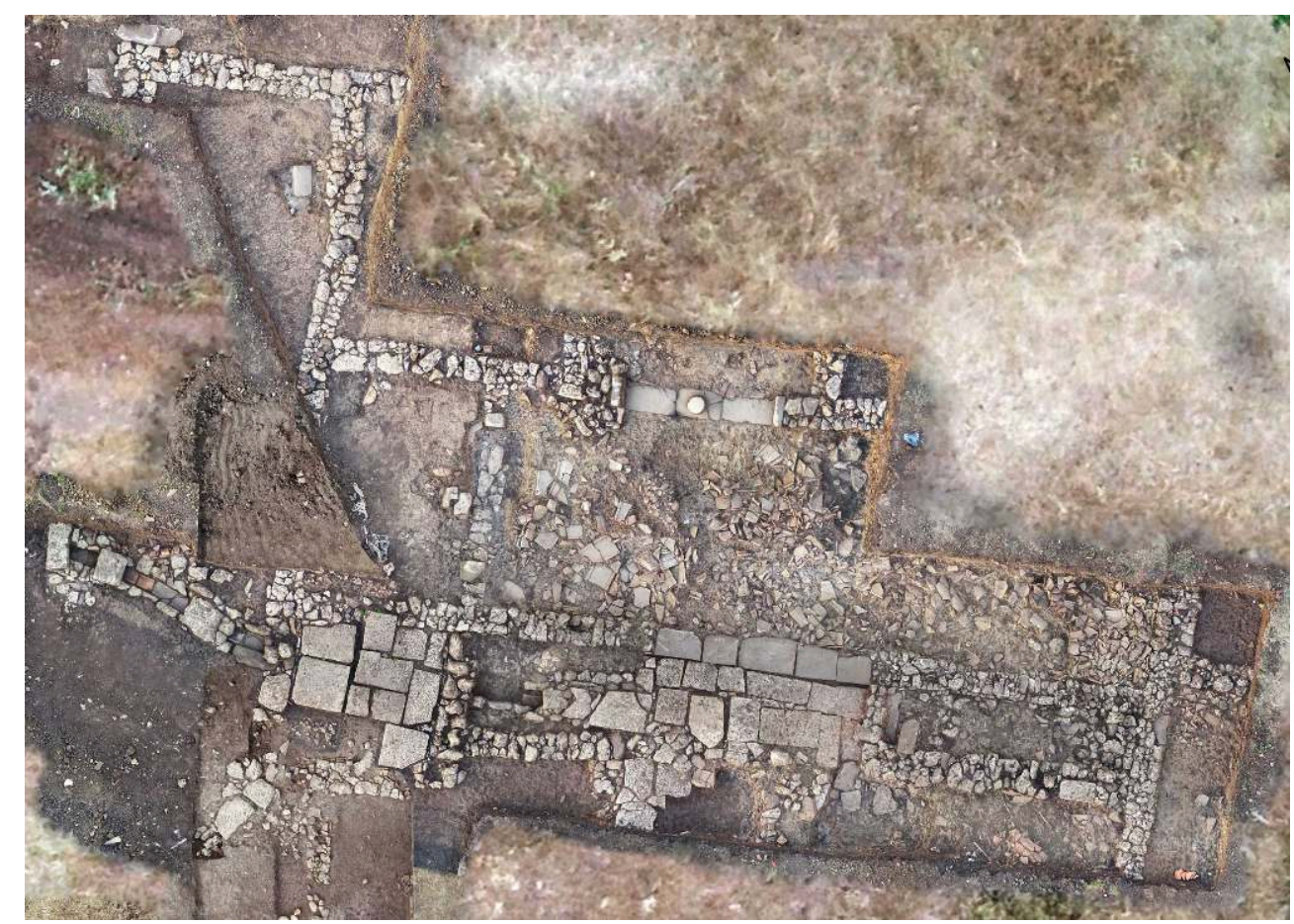

Orthophotographie T. Terrasse.

En correspondance du seuil, à l'extérieur de celui-ci se trouvait un dallage de calcaire 7202, composé en réalité de deux parties: la couverture de la canalisation 7259, est-ouest, faite de grandes dalles rectangulaires disposées longitudinalement ou transversalement; et une rangée de dalles plus étroites entre le seuil et la canalisation. 
La canalisation 7259, soigneusement construite, avec des parois latérales de petits blocs de calcaire et de grès et un fond de tuiles plates réapparaît au-delà du dallage 7160 (salle d'eau 5 : voir plus bas) et porte alors le $n^{\circ} 7264$. Le comblement 7260 et 7244 de la canalisation 7259 a été en partie fouillé. 7244 contenait, entre autres, un bord de skyphos et un fond de coupelle concavo-convexe.

Le dallage 7202 était recouvert par endroits d'un "crollo" lâche (7201 et 7238) de tegulae et d'imbrices (ainsi que d'un fragment de tuile à opaion, et d'autres de panses et bords de pithoi) qui n'est pas autre chose que le prolongement à l'extérieur de la strate d'effondrement de tuiles creuses et plates qui recouvre, plus abondamment, le sol de la pastas. C'est là une preuve supplémentaire, s'il en était besoin, que la pastas était bien entièrement couverte. Sa toiture "mixte » (avec alternance de tuiles creuses et plates) a :

- en partie chuté à la verticale (et cela vaut pour les éléments les plus pesants, les tegulae, dont certaines, presque entières, se sont déposées horizontalement sur le sol de terre battue brun gris de la pastas, au centre de celle-ci : ce crollo porte le $n^{\circ} 7230$ ) ;

- en partie s'est accumulé - comme il est normal - aux angles de la pièce : le «crollo » dense 7229, de tuiles creuses essentiellement, s'amoncelle contre l'angle sud-est de la pastas;

- en partie a glissé vers l'avant, donc juste devant la façade (et cela vaut pour des éléments plus petits et fragmentés : c'est le « crollo » 7201 et 7238).

En façade de la maison Z2, la strate 7201 recouvrant la démolition proprement dite a donné un peu de céramique surpeinte dite de Gnathia (dont un bord de bol à anses peintes, III ${ }^{\mathrm{e}}$ s. av. J.-C.), en vernis noir des coupelles concavo-convexes et monoansée, des bords et des fonds de skyphoi, des fragments d'assiette/couvercle à bourrelet et de bols à bord pendant (ces dernières formes pouvant descendre jusqu'au dernier quart du $\mathrm{III}^{\mathrm{e}} \mathrm{s}$. av. J.-C.) ; on a aussi une abondante céramique commune à pâte claire et de la céramique de cuisine, dont une grande olla à lèvre plate (7201.11), écrasée sur place en nombreux fragments. De la même strate 7201 (et du décapage 7198, qui la recouvrait) proviennent de grands fragments d'au moins trois plateaux de tables (plutôt que des louteria, avec lesquels on les confond souvent), à fond et bord plat, ornés de motifs de chevrons, de diamètre similaire $(90-100 \mathrm{~cm})$. Le démontage du «crollo " proprement dit (7238) a également livré une céramique abondante : 1 unique petit fr. de céramique à $f$. r., des bords de lékanés, de bols et d'assiettes en vernis noir, de la céramique commune et de cuisine, des balsamaires, 3 pesons, $1 \mathrm{fr}$. de statuette (chevelure ?), ainsi qu'un fragment de probable amphore cnidienne dont on parlera plus loin.

La strate d'effondrement de tuiles 7201-7238 a été nettoyée, photographiée et mise en plan avant d'être démontée soigneusement pour mettre en évidence le dallage 7202 sous-jacent. Au contraire, le « crollo » 7229-7230 a été laissé en place après nettoyage.

$\mathrm{Au}$ nord de la pastas 1 se trouvent trois pièces $(2,3$ et 4$)$ de dimensions inégales. Au milieu, la pièce $3(4,63 \times 5 \mathrm{~m})$ s'ouvre de presque toute sa largeur sur la pastas, à travers un seuil 7250, large de $3,1 \mathrm{~m}$, fait de 3 dalles de grès. Un tambour de colonne sans base (diam. $30 \mathrm{~cm}, \mathrm{~h} .30 \mathrm{~cm}$ ) était encore en place sur la dalle centrale. Deux piédroits, également en grès, délimitaient la baie de part et d'autre. Cette pièce, entièrement ouverte sur l'un de ses côtés et non susceptible d'être fermée, peut être appelée une exèdre, comme celle de la maison $\mathrm{M}$ de Civita di Tricarico, ou encore celle des maisons à péristyle de Monte Iato ou de Monte Sannace (Cazanove Féret Caravelli 2014, p. 69-71, et fig. 60). Dans la maison Z2, sur le seuil-stylobate de l'exèdre ne s'élève toutefois qu'une colonne. Par ailleurs, même si les deux seuils de la maison M (celui de la pastas 
et celui de l'exèdre) sont légèrement décalés l'un par rapport à l'autre, ils le sont beaucoup moins que dans la maison Z2. L'exèdre 3, comme dans les cas suscités, servait de pièce de desserte, d'antichambre aux deux pièces latérales 2 et 4 . C'est évident pour la pièce 2 , qui ne communiquait pas directement avec la pastas, comme le montre le fait que le mur 7213, mis au jour sur toute sa longueur à l'ouest du seuil de l'exèdre, ne présentait pas d'ouverture. Ce ne peut être que supposé pour la pièce 4 , dont seul l'angle sud-est a été mis au jour.

31 Cette partie de la maison Z2 n'a été dégagée que très partiellement, et seulement jusqu'au niveau d'arasement des murs, tandis qu'on a choisi de ne pas descendre dans les différentes pièces et donc de ne pas entamer leur stratigraphie. Le décapage superficiel de $4 \times 11 \mathrm{~m}$ qui a permis de mettre au jour le mur 7213 et le seuil 7250 porte le $n^{\circ} 7248$. Le mobilier est relativement abondant (vernis noir, dont des anses de skyphoi et un vase miniature ; céramique commune et de cuisine ; un peson). On signalera en particulier une statuette féminine debout, cassée en de nombreux morceaux, qui relève du filon tanagréen (7248.3).

La pièce 2, à l'ouest de l'exèdre et qui n'était donc desservie que par elle, a pour dimensions internes restituables 4,65 x 4,85 m. Elle est par conséquent presque carrée. On pourrait proposer, de manière très hypothétique, qu'il se soit agi d'un andrôn, d'une salle de banquets masculins couchés, pour 7 klinai. Quant à la pièce 4 , ce devait être la plus grande de toutes, pastas exceptée, avec pour dimensions restituables 6,87 x 4,85 m. C'était alors peut-être la grande pièce à vivre (oikos), à moins qu'il ne faille (beaucoup moins probablement) restituer une cage d'escalier à côté d'une pièce 4 moins grande.

L'espace 6 se trouve à l'ouest de la maison Z2. Il est délimité, comme on l'a déjà dit, par des murs antérieurs $(7223,7222,7262)$, de première phase. Mais il est toujours fonctionnel. Il ne doit pas s'agir d'une pièce fermée, comme le suggère le fait qu'on ne retrouve pas (semble-t-il) la prolongation du mur 7262 au-dessus de la canalisation 7264, et aussi l'absence de diviseur(s) partant vers l'ouest du mur ouest de Z2 (7181=7223). On a donc plutôt affaire à une galerie ouverte, peut-être un portique. L'US 7221, au-dessus du mur 7222, a livré un lot de céramique très varié, allant de l'anse d'un possible cratère à colonnettes à des fragments plus tardifs dont on parlera ci-dessous. Dans l'espace 6 a été également trouvé une base de meule de type olynthien, en pierre volcanique.

\section{Restructurations limitées de la maison Z2 (troisième phase)}

L'extension la plus visible de la maison Z2 est l'ajout d'une pièce carrée (5), de 2,9 m de côté (dim. internes) qui fait saillie sur la façade, à gauche du seuil d'entrée. Elle est délimitée par les murs 7180, 7181, 7177 et 7200. Plus que ses murs, c'est toutefois son pavement qui permet de la définir, un dallage 7160 fait de neuf grands blocs parallélépipédiques de calcaire blanc, avec - apparemment - une lacune de $2 \times 0,9 \mathrm{~m}$ dans l'angle sud-ouest. Cet espace avait été mis partiellement en lumière à la fin de la campagne 2018, mais avait alors été interprété par défaut, en l'absence de vision d'ensemble de la structure, comme un trottoir précédant la façade. Il est désormais possible d'y reconnaitre un espace fermé. Le dallage massif 7160, le fait qu'on est sur le parcours de trois canalisations, pourraient induire à interpréter cet espace comme un bassin plaqué contre le mur d'une maison. En réalité cependant, la position de l'une des dalles du pavement (qui avait déjà attiré notre attention en 2018), en quelque sorte à 
moitié à cheval sur le mur 7180, doit faire préférer une autre solution. La position de cette dalle "mordant » sur le mur, au milieu du côté nord de la pièce, montre qu'il y avait là une porte. Par conséquent, cette pièce liée à l'eau était accessible de plain-pied depuis l'intérieur de l'habitation, et c'est donc une salle de bains. La lacune apparente dans l'angle sud-ouest du pavement 7160 était sans doute l'emplacement de la baignoire. Le départ de canalisation 7188 qui pouvait servir à en évacuer l'eau, précisément de cet angle sud-ouest de la pièce 5 , conforte cette hypothèse. Il est à noter que 7188 se dirige vers la canalisation qui passera ensuite sous l'opus signinum de la salle de bains de la maison du monolithe en évacuant ses eaux usées.

Un bon parallèle pour la pièce 5 de Civita di Tricarico est constitué par la salle de bains dallée de l'édifice dit «au mundus» d'Artena (où un classique ensemble cuisine / salle d'eau a été faussement interprété comme une fosse de fondation et un autel). Les dimensions ne sont qu'un peu plus petites $(2,8 \times 2,2 \mathrm{~m})$, et on y note la présence d'une vasque ovale peu profonde. Ce parallèle avec les salles de bains d'olynthe et de Tricarico avait déjà été invoqué dans Tricarico $I^{3}$. Il se caractérise en effet, lui aussi, par la proximité entre la cuisine (avec son foyer, indispensable pour obtenir eau chaude et braises) et le bain, avec une porte de communication entre les deux. En ce sens, la cuisine devient une sorte d'antichambre de la salle de bains. On peut alors se demander si la pièce 1 bis ne serait pas, justement, une cuisine. Elle est créée dans la phase de restructuration de la maison $\mathrm{Z2}$, avec l'érection d'un mur transversal 7235 , percé d'un large seuil 7254 encadré par deux piédroits de grès distants de 2,75 m l'un de l'autre. On peut éventuellement supposer l'existence d'une colonne centrale disparue. On possède néanmoins trop peu de données sur cette pièce 1 bis pour être affirmatif sur sa fonction.

Par ailleurs, les transformations de la maison Z2 aident à comprendre celles que subit la maison Z1 qui sont tout à fait comparables. En effet, la pastas de la maison Z1 est, elle aussi, divisée dans un second temps en deux parties inégales pour créer la petite pièce 1 bis. En correspondance de celle-ci, contre la façade de l'habitation, est plaquée la pièce 4 qui reçoit un pavement de tuiles plates en remploi. Une canalisation traverse le mur sud et permet l'évacuation des eaux usées. Cette pièce 4 est sans doute la salle de bains de Z1, et la pièce 1 bis peut-être la cuisine. L'ajout d'une salle de bains, qui ne faisait pas partie du plan originel, est une caractéristique récurrente des maisons de Tricarico. C'est le cas pour la maison du monolithe ${ }^{4}$.

De l'autre côté du seuil 7203 (à l'est), se trouvent les restes d'une structure difficilement interprétable, en forme de rectangle allongé $(5,55 \times 1,85 \mathrm{~m})$ plaqué contre le mur de façade 7180 . Son côté ouest 7263 repose sur une dalle du pavement 7202 . Son côté sud est constitué par le mur probablement antérieur 7241, son côté est par le prolongement vers le sud du mur 7211. C'était donc vraisemblablement une large banquette remplie d'un blocage de moellons bruts, conservé seulement par endroits, dont le démontage a pu créer un « effet de mur » (7246) qui n'est sans doute qu'illusoire.

Quelques trouvailles permettent d'abaisser la chronologie de l'occupation de la maison Z1 (ou du moins d'une fréquentation, même réduite, du secteur) jusqu'à la fin du $\mathrm{III}^{\mathrm{e}}$ s., voire du $\mathrm{II}^{\mathrm{e}}$ s. av. J.-C.

39 Au sud du dallage 7202 qui précède le seuil de la maison Z2, en profondeur, la strate 7240 , contenait un peu de céramique (part. un fond de coupelle monoansée) et une semuncia de Rome (Mercure /proue) datable de 217-215 av. J.-C. (Crawford RRC 38/7). Même s'il s'agit peut-être d'un comblement de fosse ou d'un remblai limité dans une 
dépression (la stratigraphie, en limite sud de fouille, n'est pas parfaitement claire), la découverte de cette monnaie dans une strate non superficielle indique clairement que la maison n'est pas abandonnée avant la fin du $\mathrm{III}^{\mathrm{e}} \mathrm{s}$. av. J.-C. et la deuxième guerre punique, voire après.

Dans l'US de démolition 7201 au contact du dallage 7202, les 2 fragments les plus diagnostiques appartiennent à la panse et au départ de l'anse d'une amphore à pâte rosée peu dépurée revêtue d'engobe blanc et portant un timbre à tête de bœuf. Il devrait s'agir d'une amphore cnidienne « récente $»^{5}$. La bibliographie récente et la base "Amphoralex » du Centre d'Études Alexandrines confirment une datation à la fin II ${ }^{\mathrm{e}}$ début $\mathrm{I}^{\mathrm{er}}$ s. av. J.-C. S'il en est ainsi, l'abandon définitif du secteur de la maison Z2 intervient bien plus tard que ce à quoi on aurait pu s'attendre.

41 Dans l'espace 6, l'US 7221, au-dessus du mur 7222, a également livré 2 possibles fragments de céramique à pâte grise. Si cette identification était avérée, on aurait là un autre signe discret de la fréquentation du secteur dans la deuxième moitié du II ${ }^{\mathrm{e}} \mathrm{s}$. av. n. è.

\section{La tranchée}

Cette longue tranchée de $58 \times 2 \mathrm{~m}$, strictement orientée nord-sud, est à peu près parallèle à la tranchée de $40 \mathrm{~m}$ de long (x 2,75 m de large) qui avait été conduite en 1996 vers le nord depuis la maison des moules, et qui n'avait révélé aucune structure au-delà d'un mur léger 4282.

La tranchée 2020 était destinée à reconnaître la limite nord du quartier 3M, à vérifier qu'il n'y avait pas d'habitation au-delà de la maison Z2, à mettre en évidence d'éventuelles traces de travaux agricoles. Elle avait été planifiée pour aboutir à la structure quadrangulaire $\mathrm{Z} 5$ très visible sur la carte de prospection géophysique, dans laquelle pénétrait une canalisation. La fouille a permis de retrouver la canalisation, mais pas la structure quadrangulaire.

À $4,5 \mathrm{~m}$ au nord du mur nord de la maison $\mathrm{Z} 2$ est apparue une structure légère, dont trois murs seulement, en cailloux de petit module, sont connus. Elle mesure 7,7 $\mathrm{m} \mathrm{du}$ nord au sud (6,6 m dim. int.), sa longueur est-ouest est inconnue. Étant donné son plan, il ne s'agit vraisemblablement pas d'une habitation, peut-être d'un enclos ou encore d'un bâtiment d'exploitation.

Plus au nord, et sur $27 \mathrm{~m}$, on ne rencontre plus aucune structure. La stratigraphie, très simple, a pu être particulièrement observée dans un sondage test, de $3,2 \mathrm{~m}$ de long x $2 \mathrm{~m}$ de large x 1,3 m prof. max. (paroi est). Au-dessous de la couche de terre végétale brune, épaisse d'une cinquantaine de $\mathrm{cm}$, apparait une strate brun noir gras, qui semble vierge. Elle est épaisse d'une quarantaine de $\mathrm{cm}$. Elle repose directement sur le substrat d'argile marneuse blanc jaune. Celui-ci est donc situé beaucoup trop profond pour avoir été éraflé par la charrue, et par conséquent, on ne peut voir de traces de travail de la terre.

Plus au nord, le substrat remonte progressivement. Ce n'est sans doute pas un hasard si la dépression où la couche de la terre arable était la plus épaisse a été réservée, sans être jamais construite.

À $52 \mathrm{~m}$ de l'extrémité sud de la tranchée a été rencontrée la canalisation 7216. Elle a pu être observée sur $8 \mathrm{~m}$ de long (avec une lacune et une légère inflexion entre les deux 
tronçons conservés), après quoi elle disparaît en berme (du côté est), tandis qu'elle s'interrompt à l'ouest non loin d'une fosse (?) remplie de blocs. Les parements, de moellons de calcaire et de grès, sont conservés, mais pas les dalles de couverture. Le fond ne présentait pas d'aménagement particulier. Le canal avait une douzaine de $\mathrm{cm}$ de large, pour une largeur hors tout de la canalisation de 50-60 cm.

La canalisation 7216 reposait sur le comblement 7218 d'un creusement ovale (ou plutôt bilobé) 7217, très régulier, d'env. 5,4 (est-ouest) x $7 \mathrm{~m}$ (nord-sud) (fig. 8). La terre brun noir de 7218 contraste fortement avec l'encaissant marneux jaune blanchâtre 7219. Au sommet du comblement, des moellons désorganisés provenaient du démontage de deux structures sous-jacentes, l'embouchure 7258 d'un puits étroit (son diamètre interne ne dépasse pas $50 \mathrm{~cm}$; hauteur de la «margelle »: 911,67 m) et un massif de calage en moellons et petits blocs enserrant un bloc allongé de calcaire, de dimensions plus grandes. On propose de l'interpréter comme la base d'un balancier (tolleno, chadouf) pour tirer l'eau du puits. Ce dispositif est fréquemment associé à une canalisation, permanente ou mobile, où l'on verse l'eau pour irriguer les champs. L'embouchure du puits ne se trouve pas au centre de l'ovale régulier dessiné par le comblement 7218 . Toutefois, il ne fait pas de doute que le creusement en entonnoir 7217 qui se restreint progressivement en profondeur était destiné à l'implantation du puits et de son cuvelage de moellons de calcaire et de grès.

Fig. 8. Civita di Tricarico : le puits 7258.

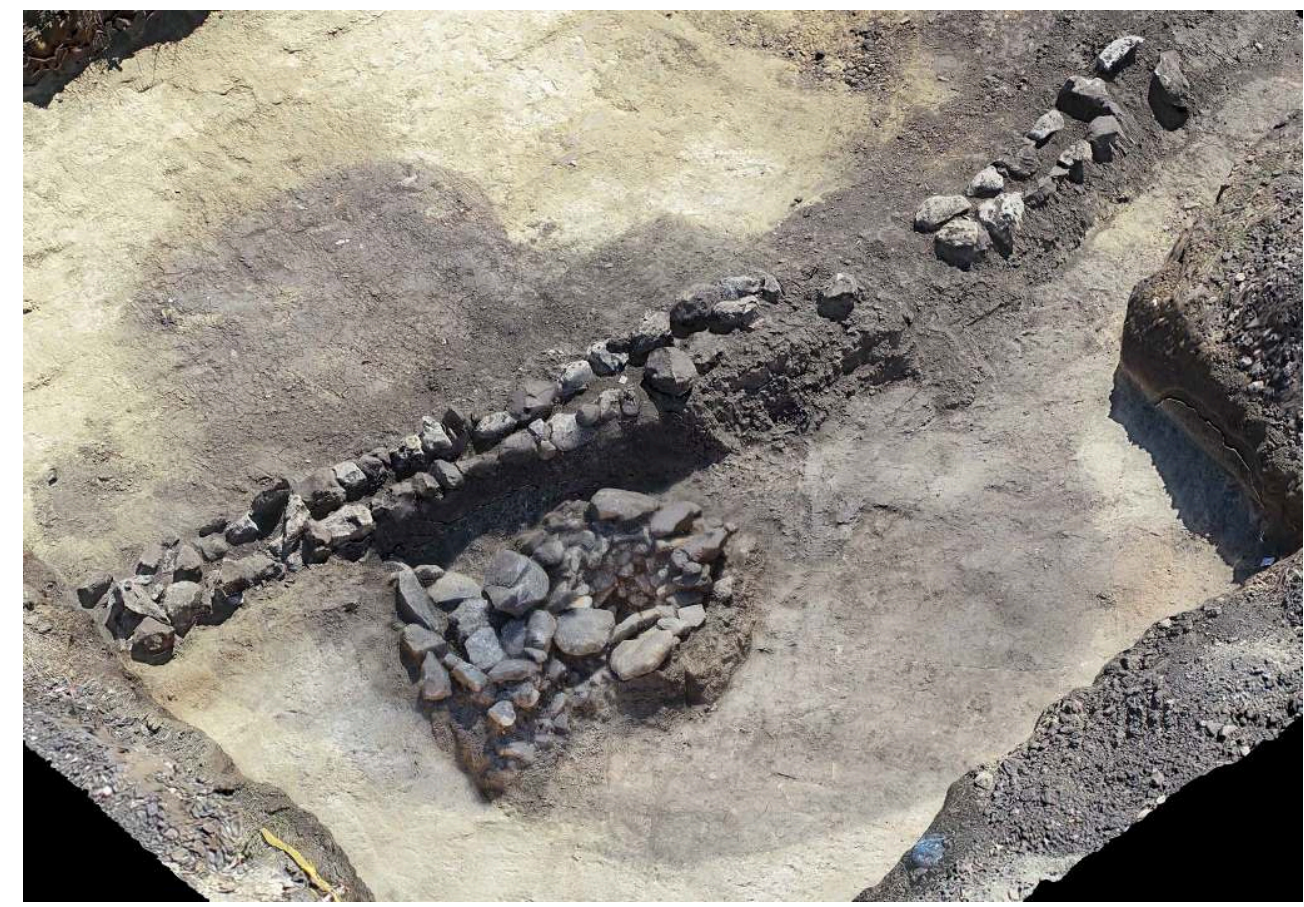

Photogrammétrie T. Terrasse.

La profondeur du puits 7258 n'est pas connue. Son diamètre diminue à mesure qu'on descend, tandis que le cuvelage a tendance à s'épaissir (fig. 9). Il a été fouillé de l'extérieur, en respectant des paliers de sécurité, sur 2,67 m de prof. (de 911,67 à 909 m s.l.m.). Plusieurs strates de comblement ont été distinguées à partir de l'orifice :

- 7220 est la couche d'effondrement sommital, contenant des moellons tombés de la margelle, pris dans une argile grise avec des inclusions calcaires, avec quelques tessons (un bord de 
tasse monoansée au vernis noir presque entièrement disparu ; 2 fragments de céramique commune et 1 fragment de cuisine ; 1 peson cassé avec un timbre ovale ;

- 7249 a une matrice argilo-sableuse gris foncé, avec de nombreuses inclusions calcaires et quelques moellons. Elle contenait un peu de mobilier et de rares fragments de tuiles ;

- 7257 est la couche inférieure du comblement fouillé. Elle se compose d'argile noire grasse et humide. Un peson (?) circulaire en terre crue grise en provient, avec plusieurs impressions de pinces à épiler et d'autres signes.

Fig. 9. Civita di Tricarico : le puits 7258 en cours de fouille.

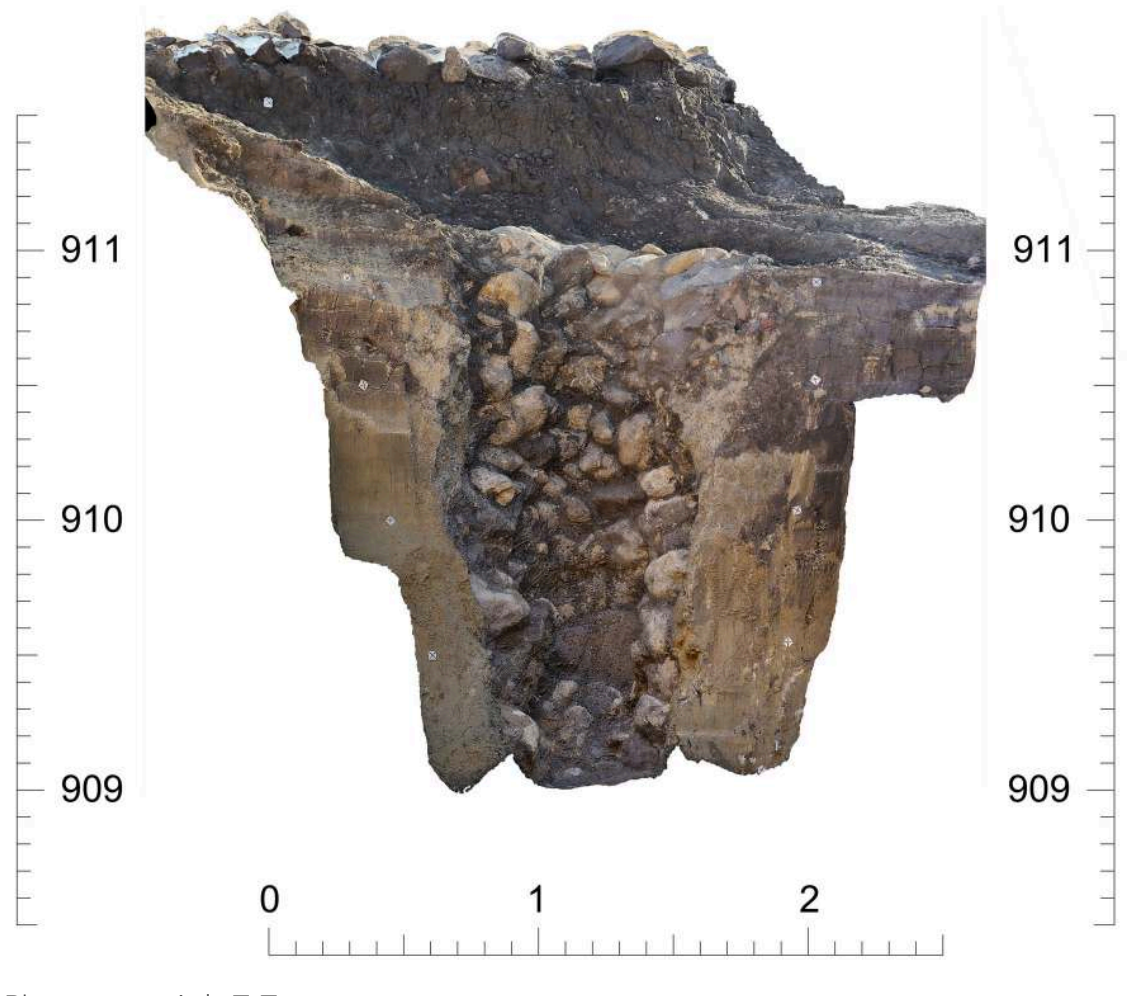

Photogrammétrie T. Terrasse.

Pour essayer de retrouver la structure Z5, ainsi que la poursuite vers l'ouest de la canalisation 7216, l'extrémité nord de la tranchée a été élargie dans cette direction, jusqu'à former une nouvelle branche longue de $15 \mathrm{~m}$ environ, large de 5,5 $\mathrm{m}$ en moyenne. Cette recherche s'est révélée vaine (ce qui est difficilement compréhensible, tant Z5 apparaissait nettement sur la prospection géophysique). En revanche, une nouvelle strate brun gris est apparue, similaire à 7218, sous la couche de décapage 7214 . $\mathrm{Au}$ centre de cette extension ouest de la tranchée, un conglomérat 7215 de blocs et de dalles calcaires, mêlé à une abondante céramique à vernis noir, commune et de cuisine, ainsi qu'à de nombreux os de bovins présentant des traces de découpe, affectait une forme grossièrement circulaire. Il s'agit à l'évidence du comblement d'une fosse, apparemment à l'intérieur d'une fosse plus grande. L'hypothèse la plus économique est alors de considérer qu'il s'agit de l'aménagement d'un puits, ensuite démonté, semblable à 7258 et antérieur à lui. On notera en tout cas que 7215 se trouve dans l'axe du prolongement de la canalisation 7216. Le trajet de celle-ci est encore indiqué, en surface, par une très légère dépression, imperceptible à l'œil nu, mais visible sur le MNT obtenu par le Lidar. Cette dépression mène à l'un des points les plus bas du site 
(le vallon de la Carnaleta excepté), qui est parfois inondé, et que la canalisation pouvait donc alimenter en eau.

\section{Bilan et perspectives}

51 En conclusion, la campagne 2020 à Civita di Tricarico a permis de documenter l'articulation entre quartier bâti et espaces libres de constructions, à vocation agricole. C'était la première fois qu'on fouillait volontairement ceux-ci. Jusque-là, en 1996 notamment, ils avaient été repérés en quelque sorte par défaut, lorsqu'on recherchait des bâtiments et qu'on n'en trouvait pas; puis, à partir de 2007-2008, on avait commencé à en cerner les limites, grâce à la prospection géophysique, mais sans vérification sur le terrain. La fouille de 2020 représente donc la première tentative pour pénétrer au cœur de ces espaces "vides ", en comprendre l'organisation et la fonction. À vrai dire, la tranchée sud-nord de $58 \mathrm{~m}$ de long ne devait être, dans le projet initial de la campagne, qu'une première étape avant le décapage d'une vaste open area permettant d'observer, à la superficie du substrat marneux blanc jaune, des traces éventuelles d'aménagement et de travail cultural (clôtures, labours, etc.). Mais cet objectif s'est très vite révélé impossible à réaliser, sur la plus grande partie du secteur traversé par la tranchée, pour une raison qu'on ne pouvait prévoir : l'abaissement très net du substrat, recouvert par 1,25 m de terre en moyenne (alors que le niveau de circulation n'a pas dû changer, comme le montrent les sols de maisons au sud, la canalisation et le puits au nord), par conséquent trop profond pour avoir été perforé par des trous de poteaux ou éraflé par les labours. Pour cette raison, le projet d'une aire ouverte en correspondance de la partie médiane de la tranchée a été abandonné.

En revanche, le niveau du substrat remonte vers le nord de la tranchée, où a pu être observé, en superficie de celui-ci, le creusement ovale d'installation du puits. Par conséquent, si l'on veut découvrir d'autres traces d'aménagement, c'est plus au nord encore qu'il faudra chercher. Pour cette raison, on peut envisager de prolonger en 2021 la tranchée commencée cette année, jusqu'au muret rectiligne de soutènement de l'agger de l'enceinte.

53 Par ailleurs, la fouille de la maison Z2 n'est pas achevée. Certes, il avait été prévu de ne la mettre en lumière que partiellement, pour en reconnaître le plan, ce qui a été effectivement fait : le plan, au moins du bloc central de l'habitation (pastas + pièces du fond), est désormais parfaitement clair. Toutefois, les niveaux de «crolli » n'ont pas encore été enlevés. Or, la durée d'occupation de la maison est un problème ouvert, comme le suggèrent certains éléments de datation plus tardifs qu'attendu. Un complément d'enquête sur la maison Z2 s'impose donc, au moins pour atteindre, dans la pastas et l'une des pièces du fond, les niveaux de sol (voire du substrat dans un sondage limité). 


\section{BIBLIOGRAPHIE}

CAZANOVE 2008

Olivier de Cazanove, Civita di Tricarico. I. Le quartier de la maison du monolithe et l'enceinte intermédiaire, Coll. de l'École Française de Rome, 409, Rome, École française de Rome, 2008.

CAZANOVE, BOURDIN 2019

Olivier de Cazanove, Stéphane Bourdin, «Ignobilia oppida Lucanorum. Recherches sur les « sites sans renom » des Lucaniens de l'intérieur (Serra del Cedro, Civita di Tricarico, habitats et sanctuaires avoisinants) », in Olivier de Cazanove, Alain Duplouy (éd.), La Lucanie entre deux mers. Histoire et archéologie (actes du colloque, novembre 2015), coll. du Centre Jean Bérard, 50, Naples, Centre Jean Bérard, 2019, p. 311-336.

CAZANOVE, FÉRET, CARAVELLI 2014

Olivier de Cazanove, Sophie Féret, Anna Maria Caravelli, Civita di Tricarico. II. Habitat et artisanat au centre du plateau, Coll. de l'École française de Rome, 483, Rome, École française de Rome, 2014.

\section{NOTES}

1. CAZANOVE, BOURDIN 2019, p. 323, fig. 14.

2. CAZANOVE, FÉRET, CARAVELLI 2014, p. 64-65, fig. 55.

3. CAZANOVE 2008, p. 113, fig. 83.

4. CAZANOVE 2008, p. 111-120 et 266-269.

5. V. GRACE BCH 1952, p. 584-585, pl. XX, 10, datée du début du $\mathrm{I}^{\mathrm{er}}$ s. av. J.-C.

\section{INDEX}

\section{Année de l'opération : 2020}

Thèmes : EFR

sujets https://ark.frantiq.fr/ark:/26678/pcrtMVyOncjILc, https://ark.frantiq.fr/ark:/26678/ pcrtSxe2PZMWR8, https://ark.frantiq.fr/ark:/26678/pcrtPPPS9ErMrW, https://ark.frantiq.fr/ ark:/26678/pcrty05M9SVnLu

lieux https://ark.frantiq.fr/ark:/26678/pcrtKwEDgM6dJk

\section{AUTEURS}

\section{STÉPHANE BOURDIN}

Université de Lyon 2 - CNRS - UMR 5189 HiSoMA 


\section{OLIVIER DE CAZANOVE}

Université Paris 1 - Panthéon Sorbonne - CNRS UMR 7041 ArScAn 